\title{
Non-motor Factors Associated with the Attainment of Community Ambulation after Stroke
}

\author{
Milene Silva Ferreira, PhD; Therezinha Rosane Chamlian, PhD; Carolina Nunes França PhD; \\ and Ayrton Roberto Massaro PhD
}

\begin{abstract}
Objective: Detect the main predictive non-motor factors related to independent community ambulation after stroke. Furthermore, we propose a scale to estimate the probability of a stroke patient achieving independent community ambulation after 6 months of rehabilitation.

Design and Settings: Prospective cohort. Subjects treated in a rehabilitation center in a large metropolitan area. Independent community ambulation was evaluated after rehabilitation according to the Hoffer classification. Functional ambulation was assessed at four levels: nonambulatory, nonfunctional ambulation, household ambulation, and community ambulation.
\end{abstract}

Participants: Patients $(\mathrm{n}=20 \mathrm{l})$ with a moderate disability after stroke.

Results: The average time of hospitalization was 19.3 days. However, only $32.8 \%$ of the patients started the rehabilitation program during the first 6 months after stroke. We found that 121 patients achieved community ambulation (60.2\%), 40 achieved household ambulation (19.9\%), 12 achieved therapeutic ambulation (5.9\%), and 28 were non-ambulatory after 6 months of treatment. Based on our final model, a scoring scale was created in order to evaluate the probability of stroke patients achieving independent community ambulation after 6 months of rehabilitation. Higher scores were associated with better chances of community ambulation within 6 months.

Conclusions: The scale that evaluated these factors proved to have acceptable sensitivity and specificity to establish the prognosis of community ambulation after 6 months of rehabilitation.

Keywords: Community ambulation; Non-motor factors; Rehabilitation; Stroke

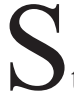
third cause of death in the United States, and the first in some developing countries. ${ }^{1,2}$ Therefore, the cost of this disease is extremely high, affecting both the individual and society. ${ }^{2,3}$ Any treatment should improve the functional outcome and must start from the early phase in specialized units with integrated services that include a rehabilitation program. ${ }^{3-5}$

In the last decade, there have been major changes in the management of patients with stroke. The disease began to be considered as a medical emergency, and treatment in intensive or even in specialized units was deployed. ${ }^{4}$ The

Corresponding Author: Milene Silva Ferreira, PhD; Alameda dos Jurupis 777, apto 242; CEP 0408800I; Brazil; Tel: 55II 993448697; Email: carolufscar24@gmail.com evolution of medical imaging and the definition of management protocols in the acute phase were also useful achievements. Thus, reduction in mortality in this population was observed; however, on the other hand, the number of people with disabilities has increased. ${ }^{6}$

Rehabilitation treatment from a multidisciplinary evaluation, covering the physical, cognitive, emotional, environmental, family history, and clinical domains of life, is highly recommended for stroke patients. ${ }^{7}$ However, the rehabilitation plan depends mainly on the prognosis, and in this area the literature is still poor.
Received: March 4, 2014

Revised: May 13, 2014

Accepted: June 30, 2014 
The negative predictors of functional recovery that are often described include: total anterior circulation infarcts, presence of urinary incontinence 2 months after injury, absence of trunk control 2 months after injury, score of $<69$ on the Functional Independence Measure (FIM) at time of admission, coma in the acute phase, prolonged hospitalization, prolonged sagging, apraxia, mixed aphasia, negligence, and age. ${ }^{8-11}$ Thus, the construction of scales that provide greater objectivity in this context is necessary. Once the impact of each variable is known, the functional outcome after a stroke depends largely on the experience of the medical professional.

Recovering the ability to walk is, for most stroke patients, the first and main objective within a rehabilitation program, ${ }^{12}$ because loss of this function is the main cause of physical dependence for these patients ${ }^{13}$. Wade and colleagues ${ }^{14}$ demonstrated that $55 \%$ of stroke survivors achieve independent walking up to 3 months after the stroke, but $45 \%$ are unable to walk.

It is known that although the first 3 months correspond to the period of greatest neuroplasticity, it remains indefinitely, regardless of age. Thus, recognizing which patients need extended and costly rehabilitation treatment is necessary for better family planning and resource management.

The aim of our study was to identify the main predictive nonmotor factors related to independent community ambulation after stroke. Furthermore, we propose a scale to estimate the probability of a stroke patient regaining independent community ambulation after 6 months of rehabilitation.

\section{Methods}

We conducted a prospective cohort study with 201 stroke patients treated in a rehabilitation center in a large metropolitan area. The protocol was created based on reviewed literature in the PubMed and MEDLINE databases, using as keywords: stroke, rehabilitation, prognosis, and gait. An evaluation form was created involving predictive factors described in the literature: (a) demographic features (age and gender); (b) number of clinical co-morbidities (hypertension, diabetes, dyslipidemia, coronary or cardiac insufficiency, arrhythmia, smoking, alcohol abuse, chronic obstructive pulmonary disease, and obesity); (c) history of prior stroke or transient ischemic attack (TIA); (d) presence of cognitive findings on neurological examination (aphasia, mixed apraxia, or heminegligence); (e) topography of the stroke lesion (hemispheric or brainstem/cerebellum); (f) presence of depression (geriatric depression scale $>10$ ) or urinary incontinence; $(\mathrm{g})$ duration of hospitalization in the acute phase; and (h) time interval between the onset of stroke and the beginning of rehabilitation longer than 6 months. Additionally, for those with ischemic stroke we also evaluated the Oxfordshire stroke classification (total anterior circulation infarct, partial circulation infarct, lacunar anterior circulation infarct, posterior circulation infarct). ${ }^{8}$
The inclusion criteria were: patients with a moderate disability (modified Rankin Score $[\mathrm{mRS}]=3$ or 4 ) and age over 18 years ${ }^{15}$ with stroke diagnosis confirmed by image exams. Patients were excluded if they had one of the following conditions: time interval from the onset of stroke to beginning of rehabilitation longer than 5 years; other neurological and psychiatric disease including dementia; any clinical instability that would interfere with the rehabilitation program; deformities and amputation of lower limbs. Additionally, we excluded those with more than $20 \%$ of absences and those who presented with a recurrent stroke or TIA during the rehabilitation treatment. The study was approved by the Research Ethics Committee from Federal University of Sao Paulo, Brazil (protocol number: 0746/06).

Patients included in the rehabilitation program, which consisted of a 30-minute session twice per week for 6 months, were evaluated at the end of the rehabilitation program to determine if they had achieved independent community ambulation according to the Hoffer classification. Evaluations were performed by the same professionals providing rehabilitation therapy. Functional ambulation was assessed at four levels: nonambulatory, nonfunctional, household, and community. ${ }^{16}$

The Hoffer classification is categorized as absent/ nonambulatory [0], therapeutic [1], household [2], and community ambulation [3]. Therapeutic ambulation is characterized as the ability to walk only with assistance from other people and/or therapeutic splint features like canvas, parallel bars, and ante-brachial walker support, for example. Household is characterized as the ability to walk independently inside the house, and community is the ability to ambulate out of the home independently or with the use mobility aids such as canes. ${ }^{17-19}$

Using the Chi-square or Fisher's exact test, each variable was analyzed to determine if the favorable outcome (independent community ambulation) was achieved after 6 months in the rehabilitation program. All categorical variables with $P$-value $<0.05$ were included in a logistic regression model. Odds ratios of the final model variables were used to construct a prognostic scale for predicting independent community ambulation after stroke. The scale was analyzed regarding its accuracy by the receiver operating characteristic (ROC) curve.

\section{Results}

Patients $(n=630)$ with stroke during the study period were eligible for inclusion in the rehabilitation treatment. Patients were excluded from the analyses if they were not able to ambulate prior to the stroke (200), had no image exams (99), had other associated brain lesions (Parkinson, $n=10$; traumatic brain injury, $\mathrm{n}=6$; hydrocephalus, $\mathrm{n}=1$; Alzheimer's, $\mathrm{n}=2$; brain tumor, $n=1$; anoxia, $n=3$; polio, $n=2$; Down Syndrome, $\mathrm{n}=1$ ); had deformities of the lower limbs (7); were lower limb amputees (5); had abandoned treatment (14); had died (6); 
Table 1. Characteristics of the patients analyzed (univariate analysis).

\begin{tabular}{|c|c|c|c|}
\hline Factors & $\begin{array}{l}\text { Community } \\
\text { ambulation } \\
\mathrm{n}(\%)\end{array}$ & $\begin{array}{c}\text { Absence of } \\
\text { community ambulation } \\
\mathrm{n}(\%)\end{array}$ & $P$ \\
\hline \multicolumn{4}{|l|}{ Age } \\
\hline$<65$ years & $67(81)$ & $42(53)$ & $P<0.001$ \\
\hline$>65$ years & $54(19)$ & $38(47)$ & \\
\hline \multicolumn{4}{|l|}{ Gender } \\
\hline Female & $57(47)$ & $42(53)$ & $P=0.454$ \\
\hline Male & $64(53)$ & $38(47)$ & \\
\hline \multicolumn{4}{|l|}{ Etiology } \\
\hline Hemorrhagic & $29(24)$ & $19(24)$ & $P=0.972$ \\
\hline Ischemic & $92(76)$ & $61(76)$ & \\
\hline \multicolumn{4}{|l|}{ Hemisphere } \\
\hline Right & $60(50)$ & 39 (49) & $P=0.779$ \\
\hline Left & $61(50)$ & $41(51)$ & \\
\hline \multicolumn{4}{|l|}{ Vascular Territory } \\
\hline Taci & $10(8)$ & $26(32)$ & $P<0.001$ \\
\hline Paci & $87(72)$ & $42(53)$ & \\
\hline Poci & $14(12)$ & $10(13)$ & \\
\hline Laci & $10(8)$ & $2(2)$ & \\
\hline \multicolumn{4}{|l|}{ Localization } \\
\hline Infratentorial & $16(13)$ & $10(13)$ & $P=0.947$ \\
\hline Supratentorial & $105(88)$ & $70(87)$ & \\
\hline \multicolumn{4}{|l|}{ Early Rehabilatition } \\
\hline$<6$ months & $42(35)$ & $24(30)$ & $P=0.486$ \\
\hline$>6$ months & $79(65)$ & $56(70)$ & \\
\hline \multicolumn{4}{|l|}{ Length in Hospital } \\
\hline$<30$ days & $104(86)$ & $38(47)$ & $P<0.001$ \\
\hline$>30$ days & $17(14)$ & $42(53)$ & \\
\hline \multicolumn{4}{|l|}{ Previous Stroke } \\
\hline Yes & $11(9)$ & $28(35)$ & $P<0.001$ \\
\hline No & $110(91)$ & $52(65)$ & \\
\hline \multicolumn{4}{|l|}{ Comorbidities } \\
\hline$<2$ & $63(52)$ & $56(70)$ & $P=0.011$ \\
\hline$>2$ & $58(48)$ & $24(30)$ & \\
\hline \multicolumn{4}{|l|}{ Apraxia } \\
\hline Yes & $11(9)$ & $29(36)$ & $P<0.001$ \\
\hline No & $110(91)$ & $51(64)$ & \\
\hline \multicolumn{4}{|l|}{ Mixed Aphasia } \\
\hline Yes & $15(12)$ & $26(32)$ & $P<0.001$ \\
\hline No & $106(88)$ & $54(68)$ & \\
\hline \multicolumn{4}{|l|}{ Hemineglect } \\
\hline Yes & $8(7)$ & $11(14)$ & $P=0.09$ \\
\hline No & $113(93)$ & $69(86)$ & \\
\hline \multicolumn{4}{|l|}{ Urinary Incontinence } \\
\hline Yes & $4(3)$ & $33(41)$ & $P<0.001$ \\
\hline No & $117(97)$ & $47(59)$ & \\
\hline \multicolumn{4}{|l|}{ Depression } \\
\hline Yes & $41(34)$ & $55(69)$ & $P<0.001$ \\
\hline No & $80(66)$ & $25(31)$ & \\
\hline
\end{tabular}

TACI: Total Anterior Circulation Infarction; PACI: partial anterior circulation infarction; POCI: Posterior Circulation infarcts; LACI: Lacunar infarcts. 
Table 2. Variables that correlate with the acquisition of gait community (multivariate analysis).

\begin{tabular}{lccc}
\hline Factors & $\beta$ & OR (CI 95\%) & $\boldsymbol{P}$ \\
\hline Age $\leq 65$ years & 1.466 & $4.334(1.7-10.9)$ & 0.002 \\
Length of hospital $<30$ days & 2.073 & $7.95(3.1-20.4)$ & 0.0001 \\
Absence of depression & 1.425 & $4.16(1.8-9.4)$ & 0.001 \\
Absence of incontinence urinary & 1.473 & $4.36(1.1-17.4)$ & 0.037 \\
Absence of apraxia & 1.328 & $3.77(1.4-10.3)$ & 0.01 \\
Absence of previous stroke & 1.996 & $7.36(2.4-21.9)$ & $<.0001$ \\
Constant & -6.656 & & \\
\hline
\end{tabular}

$\mathrm{OR}=$ odds ratio $/ \mathrm{Cl}=$ confidence interval

had a new episode of stroke during treatment (12); missed more than 15 days of treatment (13); had clinical instability (20); or had incomplete protocols (27). The remaining 201 patients completed the treatment, and were included in the analyses.

Age ranged from 21 to 90 years old (mean age $=56.9$ years), and about one-third were over 65 years of age. Forty-nine percent were male, and approximately one-fifth had a prior stroke or TIA. The majority of strokes were ischemic (76.1\%) and due to supratentorial infarcts $(87.5 \%)$. Among those with ischemic stroke, $64.2 \%$ were classified as a partial anterior circulation. Many patients (59.2\%) presented with more than two clinical co-morbidities in their prior history, and depression was a frequent finding $(47.8 \%)$ in the neurological examination. Urinary incontinence was found in $17 \%$ of patients. Among the cognitive impairments evaluated at the

Table 3. Scoring scale for evaluating non-motor factors associated with community ambulation after stroke.

\begin{tabular}{|c|c|}
\hline Clinical Factors & Score \\
\hline \multicolumn{2}{|l|}{ Age } \\
\hline$>65$ years & 0 \\
\hline$\leq 65$ years & 3 \\
\hline \multicolumn{2}{|c|}{ Hospitalization $\leq 1$ month } \\
\hline No & 0 \\
\hline Yes & 7 \\
\hline \multicolumn{2}{|l|}{ Absence of depression } \\
\hline No & 0 \\
\hline Yes & 3 \\
\hline \multicolumn{2}{|c|}{ Absence of incontinence } \\
\hline No & 0 \\
\hline Yes & 3 \\
\hline \multicolumn{2}{|l|}{ Absence of apraxia } \\
\hline No & 0 \\
\hline Yes & 3 \\
\hline \multicolumn{2}{|c|}{ Absence of a previous stroke or TIA } \\
\hline No & 0 \\
\hline Yes & 6 \\
\hline Total & 0 to 25 \\
\hline
\end{tabular}

TIA: Transient Ischemic Attack onset, aphasia was found most frequently (20\%), followed by apraxia (19.9\%), and heminegligence $(9.5 \%)$.

The mean length of hospital stay was 19.3 days $(29.3 \%>30$ days). However, only $32.8 \%$ of the patients started the rehabilitation program during the first 6 months after stroke onset.

We found 121 patients achieved community ambulation (60.2\%), 40 achieved household ambulation (19.9\%), 12 achieved therapeutic ambulation (5.9\%), and 28 were not ambulatory after 6 months of treatment. In univariate analysis, the factors associated with the acquisition of community ambulation $(P<0.05)$ were age, vascular territory, length of stay, more than one cerebrovascular event, number of comorbidities, apraxia, aphasia mixed urinary incontinence, and depression (table 1).

In multivariate analysis, six independent non-motor clinical variables were associated with community ambulation after 6 months of rehabilitation: age $\leq 65$ years (odds ratio $[\mathrm{OR}]=4.33$, $95 \%$ Confidence Interval $[\mathrm{CI}]=1.7-10.9 ; P=0.002)$; length of hospital stay $\leq 30$ days $(\mathrm{OR}=7.75, \mathrm{CI}=3.1-20.4 ; P<0.0001)$; absence of depression $(\mathrm{OR}=4.16, \mathrm{CI}=1.8-9.4 ; P=0.001)$; absence of urinary incontinence $(\mathrm{OR}=4.36, \mathrm{CI}=1.1-17.4 ; P<$ 0.037); absence of apraxia (OR=3.77, $\mathrm{CI}=1.4-10.3 ; P=0.01)$; and absence of prior history of stroke or TIA (OR=7.36, $\mathrm{CI}=2.4-21.9 ; P<0.0001)$ (table 2).

Based on our final model, a scoring scale was created (scores ranging from 0 to 25 ) to evaluate the probability of stroke patients achieving independent community ambulation after 6 months of rehabilitation (table 3). We applied the scoring

Table 4. Probability for community ambulation after stroke obtained from scores derived from our patients.

\begin{tabular}{ccc}
\hline Scores & N & Community Ambulation \\
\hline $0-11$ & 23 & 0 \\
$12-13$ & 20 & $10 \%$ \\
$14-15$ & 16 & $31 \%$ \\
$16-19$ & 52 & $65 \%$ \\
$20-25$ & 90 & $89 \%$
\end{tabular}




\section{ROC Curve}

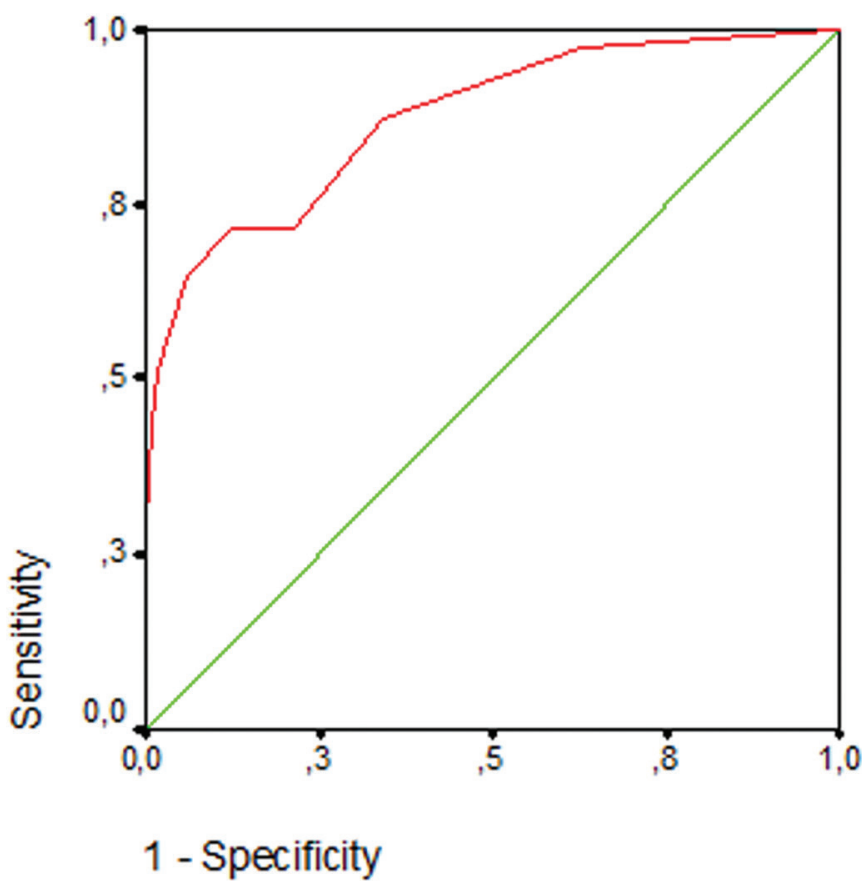

Diagonalsegments are produced by ties.

Area under Curve $=0,87(0,82$ a 0,92$), p<0.0001$

Figure 1. ROC curve showing sensitivity and specificity of the score.

scale system to our stroke cohort to verify the probability for achieving community ambulation. Higher scores were associated with higher chances of community ambulation within 6 months (table 4). The average area under the ROC curve was 0.87 (figure 1), which demonstrated good sensitivity and specificity.

\section{Discussion}

Our study evaluated the impact of non-motor aspects in the prognosis for ambulation after stroke. The population of patients studied was in chronic phase, which is scarce in the literature.

Some studies assessed the impact of changes in cognitive rehabilitation treatment of patients with stroke, and functional outcomes by the Barthel Index, modified Rankin scale, and the ability to walk by the Hoffer classification. The authors concluded that it is possible to obtain good results with a functional rehabilitation program, despite the cognitive changes. However, there are significant differences between groups with respect to the acquisition of community ambulation. ${ }^{20-22}$

Community ambulation is an important goal for a stroke rehabilitation program. ${ }^{16}$ The acquisition of this function might be influenced by several factors including walking speed, motor function, balance, endurance, and use of a walking aid. ${ }^{23}$ However, it is known that factors not related to motor function also have high impact on the acquisition of community ambulation, such as clinical, sociodemographic, cognitive, and emotional.

Considering the time for beginning rehabilitation, it is unfortunate that only $32.8 \%$ of our study population had started treatment $<6$ months after the stoke event. This wide range of time is probably the lack of focused and specialized treatment in the acute phase, the lack of medical knowledge about the importance of rehabilitation, and also the lack of specialized centers, meaning that patients need to wait a long time to begin treatment. We should consider that this is a public health problem and that the development of policies and improved treatment for stroke must be deployed.

Regarding co-morbidities, most of our population had more than two pathologies (eg, hypertension, diabetes mellitus, dyslipidemia, arrhythmias, heart failure, coronary artery disease, alcoholism, chronic obstructive pulmonary disease), demonstrating the high cardiovascular risk in this population. A stroke of the middle cerebral artery predominates over the other vascular territories, ${ }^{24}$ and in our study, this variable was confirmed.

Age has been reported by many authors as a negative predictive factor both for acquisition of independence and functional ambulation. According to Jorgensen et al, ${ }^{17}$ worse diagnosis over age 65 years is due to a reduction in patients' abilities for functional compensation and not to an increase in co-morbidities. According to Sanches-Blanco et al, ${ }^{25}$ patients under 70 years have twice the probability of achieving independent ambulation. The present study demonstrated that individuals over age 65 have four times less chance of achieving community ambulation than younger patients. A prolonged internment time means greater severity and extension of the lesion and, according to Davidoff et al, ${ }^{26}$ was related to worse results in terms of self-care and ambulation, which is compatible with our results. It is believed that besides meaning greater severity of the lesion and a prolonged hospitalization time in the acute phase, it is also associated with a higher number of complications secondary to immobilization.

Depression frequently occurs after a stroke, and the prevalence rate described ranges between $25 \%$ and $79 \%$ of cases. ${ }^{27}$ In a recent systematic review, it was estimated that one-third of the patients with stroke develop depression..$^{28,29}$ In that study, frequency of depression was high, occurring in $47.8 \%$ of patients. This is an important point to be considered, as it is known that depression is related to an increase in death rate, a delay in recovery, poor quality of life, higher rate of internments as well as worse functional indexes at the end of

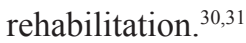

The persistence of urinary incontinence for more than 2 months after the injury is directly related to worse functional 
outcomes and the inability to walk. In this sample, $97.5 \%$ of individuals with urinary incontinence did not acquire the ability to ambulate in the community. ${ }^{32,33}$

According to Kwakkel and colleagues, ${ }^{34}$ control of the trunk in the first week post-stroke, was an independent variable for predicting comfortable ambulation at 6 months. Hama and colleagues $^{35}$ conducted a study with 452 patients, aiming to verify the association of trunk control, depressive symptoms, and functional outcomes. The authors concluded that inefficient trunk control is strongly associated with depression, deficit of initiative and apathy, and predicts a worse functional outcome. This suggests evaluating patients for depression maybe useful in planning treatments and predicting functional outcomes. ${ }^{36,37}$

Our scoring scale may be a new tool to be used for identifying stroke survivors with potential for acquisition of ambulation after a rehabilitation program. This work shows that this instrument has good specificity and sensitivity in the internal validation. However, additional studies are needed to determine its reproducibility.

\section{Conclusion}

We conclude that, from among the non-motor factors studied, those associated with the attainment of community ambulation are: age, previous stroke, internment time, ideomotor apraxia, urinary incontinence, and depression.

The evaluation scale which used these factors proved to have acceptable sensitivity and specificity (area under the curve $\mathrm{ROC}=0.87$ ) to establish the prognosis of community ambulation after 6 months of rehabilitation.

\section{Acknowledgements}

The authors thank the Federal University of Sao Paulo and Hospital Israelita Albert Einstein, Sao Paulo, Brazil for its support of this study.

\section{References}

1. Marler JR, Tilley BC, Lu M, Brott TG, Lyden PC, Grotta JC, Broderick JP, Levine SR, Frankel MP, Horowitz SH, Haley EC Jr, Lewandowski CA, Kwiatkowski TP. Early stroke treatment associated with better outcome: the NINDS rt-PA stroke study. Neurology 2000;55:1649-1655.

2. Lessa I. Epidemiologia das doenças cerebrovasculares no Brasil. Rev Soc Cardiol Estado de São Paulo. 1999;9:509-518.

3. Goldstein LB, Adams R, Alberts MJ, Appel LJ, Brass LM; Bushnell CD, Culebras A, Degraba TJ, Gorelick PB, Guyton JR, Hart RG, Howard G, Kelly-Hayes M, Nixon JV, Sacco RL; American Heart Association/American Stroke Association Stroke Council; Atherosclerotic Peripheral Vascular Disease Interdisciplinary Working Group; Cardiovascular Nursing Council; Clinical Cardiology Council; Nutrition, Physical Activity, and Metabolism Council; Quality of Care and Outcomes Research Interdisciplinary Working Group; American Academy of Neurology. Primary prevention of ischemic stroke: a guideline from the American Heart Association/American Stroke Association Stroke Council: cosponsored by the Atherosclerotic Peripheral Vascular Disease Interdisciplinary
Working Group; Cardiovascular Nursing Council; Clinical Cardiology Council; Nutrition, Physical Activity, and Metabolism Council; and the Quality of Care and Outcomes Research Interdisciplinary Working Group: the American Academy of Neurology affirms the value of this guideline. Stroke 2006;37:1583-1633.

4. The European Ad Hoc Consensus Group. Optimizing intensive care in stroke. A European Perspective. Cerebrovasc Dis 1997;7:113-128.

5. Kaste M, Skyhoj Olsen T, Orgozo JM, Bogousslavsky J, Hacke W. Organization of stroke care: education, stroke units and rehabilitation. European Stroke Initiative (EUSI). Cerebrovasc Dis 2000;10:1-11.

6. Masiero S, Avesani R, Armani M, Verena P, Ernani M. Predictive factors for ambulation in stroke patients in the rehabilitaiton setting: a multivarieta analysis. Clin Neurol Neurosurg. 2007;109:763-69.

7. Duncan PW, Zorowitz R, Bates B, Choi JY, Glasberg JJ, Graham GD, Katz RC, Lamberty K, Reker D. Management of Adult Stroke Rehabilitation Care: a clinical practice guideline. Stroke. 2005;36;100-143.

8. Stone SP, Allder SJ, Gladman JR. Predicting outcome in acute stroke. Br Med Bull 2000;56:486-494.

9. Hajek VE, Gagnon S, Ruderman JE. Cognitive and functional assessments of stroke patients: an analysis of their relation. Arch Phys Med Rehab1997;78:1331-1337.

10. Gross JC. Urinary incontinence and stroke outcomes. Arch Phys Med Rehabil 2000;81:22-27.

11. Barer DH. Continence after stroke: useful predictor or goal of therapy? Age Ageing 1989;18:183-191.

12. Bohannon MG, Wikholm JB. Importance of four variables of walking to patients with stroke. Int J Rehabil Res 1991;14:246-250.

13. Dobkin BH. Strategies for stroke rehabilitation. Lancet Neurol 2004;3:528-356.

14. Wade DT, Wood VA, Heller A. Walking after stroke. Measurement and recovery over the first 3 months. Scand J Rehab Med 1987;19:25-30.

15. Kollen B, van de Port I, Lindeman E, Twisk J, Kwakkel G. Predicting improvement in gait after stroke. Stroke 2005;36:2676-2680.

16. Hoffer MM, Feiwell E, Perry R, Perry J, Bonnett C. Functional ambulation in patients with myelomeningocele. J Bone Joint Surg Am 1973;5:137-148.

17. Jørgensen HS, Reith J, Nakayama H, Kammersgaard LP, Raaschou HO, Olsen TS. What determines good recovery in patients with the most severe strokes? The Copenhagen Stroke Study. Stroke 1999;30:2008-2012.

18. Viosca E, Lafuente R, Martínez JL, Almagro PL, Gracia A; González C. Walking recovery after an acuete stroke: assessmente with a new functional classification and the Barthel Index. Arch Phys Med Rehabil 2005;86:1239-1244.

19. Sánchez-Blanco I, Ochoa-Sangrador C, López-Munaín L, Izquierdo-Sánchez, M, Fermoso-García J. Predictive model of functional independence in stroke patients admitted to a rehabilitation programme. Clin Rehabil 1999;13:464-475.

20. van de Port IG, Kwakkel G, Schepers VP, Lindeman E. Predicting mobility outcome one year after stroke: a prospective cohort study. J Rehabil Med 2006;38:218-223.

21. Jehkonen M., Ahonen JP, Dastidar P, Koivisto AM, Laippala P, Vilkki J, Molnár G. Visual neglect as a predictor of functional outcome one year after stroke. Acta Neurol Scand 2000;101:195-201.

22. Wandel A, Jorgensen HS, Nakayama H, Raaschou HO, Olsen TS. Prediction of walking function in stroke patients with initial lower extremity paralysis: the Copenhagen Stroke Study. Arch Phys Med Rehabil 2000;81:736-738. 
23. Carod-Artal FJ, Medeiros MS, Horan TA, Braga LW. Predictive factors of functional gain in long-term stroke survivors admitted to a rehabilitation programme. Brain Inj 2005;19:667-673.

24. Murphy E. Stroke rehabilitation. J R Coll Physicians Lond 1999;33:466-468.

25. Sánchez-Blanco I, Ochoa-Sangrador C, López-Munaín L, Izquierdo-Sánchez M, Fermoso-García J. Predictive model of functional independence in stroke patients admitted to a rehabilitation programme. Clin Rehabil 1999;13:464-475.

26. Davidoff G, Keren O, Ring H, Solzi P, Werner RA. Assessing candidates for inpatient stroke rehabilitation: predictors of outcome. Phys Med Rehabil Clin NA 1991;2(3):501-516.

27. Thom T, Haase N, Rosamond W, Howard VJ, Rumsfeld J, Manolio T, Zheng ZJ, Flegal K, O’Donnell C, Kittner S, Lloyd-Jones D, Goff DC Jr, Hong Y, Adams R, Friday G, Furie K, Gorelick P, Kissela B, Marler J, Meigs J, Roger V, Sidney S, Sorlie P, Steinberger J, Wasserthiel-Smoller S, Wilson M, Wolf P; American Heart Association Statistics Committee and Stroke Statistics Subcommittee. Heart disease and stroke statistics--2006 update: a report from the American Heart Association Statistics Committee and Stroke Statistics Subcommittee. Circulation 2006;113:85-151.

28. Hackett ML, Anderson CS. Predictors of depression after stroke: a systematic review of observational studies. Stroke 2005;36:2296-2301

29. Jia H, Damush TM, Qin H, Ried LD, Wang X, Young IJ, Williams LS. The impact of poststroke depression on healthcare use by veterans with acute stroke. Stroke 2006;37:2796-2801.

30. Paolucci S, Grasso MG, Antonucci P, Bragoni M, Troisi E, Morelli D, Coiro P, De Angelis D, Rizzi F. Mobility status after inpatient stroke rehabilitation: 1-year follow-up and prognostic factors. Arch Phys Med Rehabil 2001;82:2-8.

31. Kauhanen ML, Korpelainen JT, Hiltunen P, Brusin E, Mononen H, Määttä R, Nieminen P, Sotaniemi KA, Myllylä VV. Poststroke depression correlates with cognitive impairment and neurological deficits. Stroke 1999;30:1875-1880.

32. Tapia CI, Khalaf K, Berenson K, Globe D, Chancellor M, Carr LK. Health-related quality of life and economic impact of urinary incontinence due to detrusor overactivity associated with a neurologic condition: a systematic review. Health Qual Life Outcomes 2013;11:13.

33. Turhan N, Atalay A, Atabek HK. Impact of stroke etiology, lesion location and aging on post-stroke urinary incontinence as a predictor of functional recovery. Int J Rehab Res 2006;29:335-338.

34. Kwakkel G, Kollen BJ, Wagenaar RC. Long term effects of intensity of upper and lower limb training after stroke: a randomised trial. J Neurol Neurosurg Psychiatry 2002;72:473-479.

35. Hama S, Yamashita H, Shigenobu M, Watanabe A, Hiramoto K, Takimoto Y, Arakawa R, Kurisu K, Yamawaki S, Kitaoka T. Sitting balance as an early predictor of functional improvement in association with depressive symptoms in stroke patients. Psychiatry Clin Neurosci 2007;61:543-551.

36. Wolters G, Stapert S, Brands I, Van Heugten C. Coping styles in relation to cognitive rehabilitation and quality of life after brain injury. Neuropsychol Rehabil 2010;20:587-600.

37. Singh R, Hunter J, Philip A, Todd I. Predicting those who will walk after rehabilitation in a specialist stroke unit. Clin Rehab 2006;20:149-152.

\section{Author Affiliations}

Milene Silva Ferreira, PhD ${ }^{*,+}$; Therezinha Rosane Chamlian, PhD*; Carolina Nunes França, PhD ${ }^{\S, \|}$; Ayrton Roberto Massaro, $P h D^{\natural}$

*Federal University of Sao Paulo, Sao Paulo, Brazil

${ }^{r}$ Hospital Israelita Albert Einstein, Sao Paulo, Brazil

* Physiatry Division, Federal University of Sao Paulo, Brazil

${ }^{\S}$ Cardiology Division, Federal University of Sao Paulo, Brazil

'Santo Amaro University, Sao Paulo, Brazil

"Neurology Division, Federal University of Sao Paulo, Brazil 